\title{
Estudo de Fiabilidade da Escala de Capacidade de Controlo da Diabetes: Versão Breve
}

\author{
Fiability Study of Diabetes Empowerment Scale: Short Version
}

\author{
Marcelo AVEIRO1 ${ }^{1}$, Luiz Miguel SANTIAGO²,3, Pedro LOPES FERREIRA ${ }^{4}$, José Augusto SIMÕES 5 \\ Acta Med Port 2015 Mar-Apr;28(2):177-181
}

RESUMO

Objetivo: Avaliar a correlação entre o valor obtido pelo instrumento de medição Diabetes Empowerment Scale - Short Form e o controlo da pessoa com diabetes medido pelo valor da hemoglobina glicada A1c.

Material e Métodos: Estudo observacional transversal pela aplicação do Diabetes Empowerment Scale - Short Form a pessoas com diabetes de três Unidades de Saúde Familiar da Região Centro de Portugal após realização de teste e reteste (primeiro por escrito e, passados cinco minutos, oralmente) para determinação da coerência interna através do valor de alfa de Cronbach em 20 elementos que não foram depois estudados. A aplicação a pacientes diabéticos foi feita após a consulta de enfermagem e antes da entrada na consulta médica. Foi realizada estatística descritiva e inferencial apos verificação da normalidade dos dados.

Resultados: Na primeira fase o valor de alfa de Cronbach de 0,90 a 1,00 relativamente aos oito itens da escala. Na aplicação escrita, a média de resultados foi de $3,78 \pm 0,71$ e na aplicação oral de $3,79 \pm 0,65, p=0,629$. A amostra da segunda fase foi de 81 pessoas com diabetes, sendo $55,6 \%$ do sexo masculino. A idade média foi de $68,5 \pm 1,1$ anos com uma HbA1c média de 6,8 $\pm 0,2$ e um tempo de evolução desde o diagnóstico de $9,2 \pm 0,9$ anos. A média da pontuação final da escala foi de $4,1 \pm 0,8$. Verificou-se uma correlação significativa entre a pontuação final e os níveis de Hba1C $(\rho=-0,114 ; p=0,312)$.

Conclusão: A Escala de Capacidade de Controlo da Diabetes - Versão Breve revelou ser uma escala fiável para medir a capacitação em doentes diabéticos em Portugal. Confirmou-se a presença de uma correlação estatisticamente significativa entre o resultado obtido no final da escala e o valor de HbA1c.

Palavras-chave: Diabetes Mellitus Tipo 2; Poder (Psicologia); Questionários.

\section{ABSTRACT}

Purpose: To compare the final score of the scale to the levels of HbA1c.

Material and Methods: Cross-sectional observational study by applying the Diabetes Empowerment Scale - Short Form to diabetics at three primary care units in the central region of Portugal. The test-retest (in writing first and, five minutes later, orally) was performed to access Cronbach's alpha in 20 patients not studied in the next phase. Then, the scale was applied to diabetic patients after nursing consultation and prior to entering the medical consultation. Descriptive and inferential statistics after checking for the normality of the data were performed.

Results: In the first phase Cronbach's alpha was 0.90 to 1.00 in all of eight scale items. The average result obtained in the written phase was $3.78 \pm 0.71$ and in the oral $3.79 \pm 0.64, p=0.629$. The sample of the second stage was of 81 diabetic patients, $55.6 \%$ male. Sample's mean age was $68.5 \pm 1.1$ years, mean $\mathrm{HbA} 1 \mathrm{c}$ of $6.8 \pm 0.2$ and mean time from diagnosis of $9.2 \pm 0.9$ years. The average final score of the scale was $4.1 \pm 0.8$. There was significant correlation between the final score and HbA1c levels $(\rho=-0.114 ; p=0.312)$.

Conclusion: The Portuguese version of the Diabetes Empowerment Scale - Short Form proved to be a reliable scale to measure empowerment in diabetic patients in Portugal. It was evident the presence of a statistically significant correlation between the results obtained at the end of the scale and HbA1c.

Keywords: Diabetes Mellitus, Type 2; Power (Psychology); Questionnaires.

\section{INTRODUÇÃO}

Os últimos dados do Relatório Anual do Observatório da Diabetes de novembro de 2013 revelam que a diabetes é uma doença que atinge cerca de $8,3 \%$ da população mundial. Em 2012, cerca de 12,9\% da população nacional entre os 20 e os 79 anos era afetada por esta doença. Deste universo de doentes diabéticos, cerca de $51,9 \%$ tem um valor de hemoglobina glicada (HbA1C) inferior a $6,5 \%$ e $25 \%$ tem a $\mathrm{HbA} 1 \mathrm{C}$ superior a $8 \%{ }^{1}$

De uma forma geral, em 2012 a diabetes teve um custo direto estimado entre 1250 e 1500 milhões de euros. Este valor representa $9 \%$ dos gastos totais em saúde e cerca

\section{0,9\% do PIB português em $2012 .^{1}$}

O objetivo da educação de doentes diabéticos é o aumento da sua capacidade de usar o conhecimento sobre a diabetes, adquirindo a autonomia necessária para o seu controlo nos mais diversos aspetos da sua vida diária. ${ }^{2}$ Esse controlo poderá vir a ser conseguido através da capacitação definida como 'ajudar as pessoas a descobrir e a usar a sua capacidade inata para ganhar mestria sobre a sua diabetes'. ${ }^{3}$ Já em 1991 foi realizado um estudo que teve como objetivo avaliar a efetividade de um programa de capacitação de diabetes, focando-se inteiramente em

1. Faculdade de Medicina. Universidade de Coimbra. Coimbra. Portugal.

2. Unidade de Saúde Familiar Topázio. Agrupamento de Centros de Saúde do Baixo Mondego. Coimbra. Portugal.

3. Faculdade de Ciências da Saúde. Universidade da Beira Interior. Covilhã. Portugal.

4. Faculdade de Economia. Universidade de Coimbra. Coimbra. Portugal.

5. Unidade de Saúde Familiar Marquês de Marialva. ADES Baixo Mondego. Cantanhede. Portugal.

Recebido: 17 de Julho de 2014 - Aceite: 23 de Dezembro de 2014 | Copyright $\odot$ Ordem dos Médicos 2015 
aspetos psicossociais como o controlo do stress, o suporte familiar, a negociação com profissionais de saúde e o lidar com emoções desconfortáveis. ${ }^{3}$

Devido à dificuldade encontrada em conseguir medir a autoeficácia na diabetes, definida como a capacidade de um indivíduo para se envolver em tarefas de autocontrolo em situações específicas como a monitorização de níveis da glucose e o encomendar refeições em restaurantes, entre outros, foi criada a Diabetes Empowerment Scale (DES), um questionário com 37 itens, medindo oito dimensões das quais apenas três foram depois usadas por terem a necessária consistência interna superior ou igual a 0,8. Tal estudo revelou que o programa de capacitação implementado trouxe melhorias efetivas a nível psicossocial e a nível de parâmetros laboratoriais. ${ }^{4}$

Mais tarde, de forma a permitir uma avaliação mais breve e global, foi criada uma versão mais curta com oito perguntas - a DES-SF - que, aplicada originalmente a 229 doentes, revelou uma fiabilidade de $0.84 .{ }^{5} \mathrm{~A}$ sua validade foi comprovada pelo facto de tanto as pontuações do questionário como os níveis de $\mathrm{HbA} 1 \mathrm{c}$ terem, durante 6 semanas, revelado uma mudança positiva após um programa de educação para diabéticos. ${ }^{5}$ Este questionário já foi usado com sucesso em vários programas de educação para a diabetes, com o objetivo de avaliar as componentes psicosso-

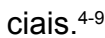

Em 2013, a DES-SF foi linguística e culturalmente adaptada para português pelo Centro de Estudos e Investigação em Saúde da Universidade de Coimbra (CEISUC). Após a devida autorização dos autores do Michigan Diabetes Research and Training Center, a versão original foi traduzida, tendo sido seguido o processo de tradução/retroversão, após o que se procedeu a uma revisão clínica e a um teste cognitivo de compreensão para garantir a boa interpretação das perguntas pelos diabéticos.

O presente estudo teve como objetivo aplicar o inquérito a diabéticos tipo 2 da Região Centro, de forma a aferir a sua fiabilidade e validade pelo teste de alfa de Cronbach e, para a população portuguesa, testar uma possível correlação entre as pontuações do DES-SF e os níveis de HbA1c.

Esta escala pode constituir-se numa importante ferramenta para a melhor percepção, pelas equipas que lidam com pessoas sofrendo de diabetes, quanto ao que ainda é necessário fazer para que as escolhas a serem diariamente feitas possam ser melhoradas.

\section{MATERIAL E MÉTODOS}

Estudo observacional transversal pela aplicação do DES-SF. A este questionário foi adicionado um documento de consentimento informado e um questionário epidemiológico, onde se inquiriu sobre o género, o valor médio dos dois últimos valores de $\mathrm{HbA} 1 \mathrm{c}$, o grau de instrução e o tempo de diagnóstico, em anos. A participação foi voluntária, anónima e confidencial. Foi obtido parecer favorável da Comissão de Ética da Administração Regional de Saúde do Centro. Nos casos em que a pessoa, sofrendo de diabetes, não sabia ler nem escrever foi pedido ao acompanhante que Ihe fizesse a aplicação do questionário.

Após, pedido e consentimento dos autores americanos por escrito, iniciou-se a fase de obtenção de uma versão equivalente sob o ponto de vista linguístico e semântico do DES-SF realizado na CEISUC, procedeu-se à aplicação do questionário a um conjunto de pessoas sofrendo de diabetes tipo 2, metade das quais sob insulinoterapia.

O estudo de campo foi realizado em duas fases em amostras de conveniência. A primeira fase consistiu na validação da fiabilidade da escala que foi aplicada a 20 doentes diabéticos da USF Marquês de Marialva, em Cantanhede, no fim da consulta de seguimento, onde, primeiramente, o preenchimento era feito pelo próprio utente e, passados cinco minutos, o entrevistador aplicava o questionário oralmente e marcava as respostas que o utente fornecia.

$\mathrm{Na}$ segunda fase a aplicação estendeu-se a mais duas Unidades de Saúde Familiar (USF), nomeadamente a Topázio e a S. Julião. O questionário foi preenchido pelo próprio doente, de novo, após a consulta de enfermagem de seguimento de diabetes em duas semanas uma do mês de janeiro e outra do mês de fevereiro de 2014 .

Foi feita a análise de estatística descritiva e inferencial com o programa SPSS versão 20.0, utilizando o teste $t$-Student para variáveis emparelhada, o $U$ de Mann-Witney, o coeficiente de correlação de Pearson e o teste ANOVA, após demonstração da normalidade dos dados contínuos.

\section{RESULTADOS}

\section{Primeira Fase}

A amostra obtida foi de 20 diabéticos, utentes da USF Marquês de Marialva em Cantanhede constituída maioritariamente por elementos do sexo feminino $(55,0 \%)$, por pessoas com frequência dos $3^{\circ}$ ou $4^{\circ}$ ano $(65,0 \%)$, com uma idade média de $71,9 \pm 12,4$ anos, apresenta média de valor das duas últimas conhecidas $\mathrm{A} 1 \mathrm{c}$ de $7,0 \pm 1,3$ e uma média do tempo desde o diagnóstico de $7,7 \pm 3,9$ anos conforme a Tabela 1.

Para o teste-reteste calcularam-se os valores de correlação de Pearson e intraclasse apresentados na Tabela 2.

A média de pontuação nas respostas foi de $3,8 \pm 0,7$ na aplicação por escrito e de $3,8 \pm 0,7$ na aplicação oral, $p=0,629$.

\section{Segunda Fase}

A segunda fase contou com uma amostra maior de 81 diabéticos, obtida na USF Marquês de Marialva, Cantanhede, USF Topázio, Coimbra e USF S. Julião, Figueira da Foz.

A Tabela 3 mostra a distribuição das variáveis género, instrução, idade, $\mathrm{HbA} 1 \mathrm{c}$ e tempo de diagnóstico. A percentagem de elementos do sexo masculino é de $55,6 \%$, o grau de instrução mais prevalente é ter o ensino básico (66,7\%). A idade média é de $68,5 \pm 10,3$ anos, o valor médio das duas últimas medições da $\mathrm{HbA} 1 \mathrm{c}$ é de $6,8 \pm 1,3 \%$ e o tempo médio desde o diagnóstico de diabetes tipo 2 de 9,2 \pm 8,0 anos.

A Tabela 4 apresenta a distribuição das frequências por 
Tabela 1 - Dados sociodemográficos e clínicos da amostra 1

\begin{tabular}{|c|c|c|c|}
\hline Variável & Valores & $\mathbf{n}$ & $\%$ \\
\hline Amostra & & 20 & 100,0 \\
\hline \multirow[t]{2}{*}{ Género } & Masculino & 9 & 45,0 \\
\hline & Feminino & 11 & 55,0 \\
\hline Idade (anos) & $\begin{array}{l}\text { Média } \pm \text { d.p. } \\
\text { IC a } 95 \%\end{array}$ & $\begin{array}{c}71,85 \pm 12,42 \\
66,0-77,7\end{array}$ & \\
\hline \multirow[t]{5}{*}{ Habilitações Literárias } & Não sabe ler nem escrever & 0 & 0,0 \\
\hline & Sabe ler e escrever & 6 & 30,0 \\
\hline & Ensino básico ( $6^{\circ}$ ano $)$ & 13 & 65,0 \\
\hline & Ensino Secundário $\left(12^{\circ}\right.$ ano $)$ & 1 & 5,0 \\
\hline & Ensino Superior & 0 & 0,0 \\
\hline Hba1c & $\begin{array}{l}\text { Média } \pm \text { d.p. } \\
\text { IC a } 95 \%\end{array}$ & $\begin{array}{c}71,85 \pm 1,31 \\
6,6-7,6\end{array}$ & \\
\hline Tempo de diagnóstico & $\begin{array}{l}\text { Média } \pm \text { d.p. } \\
\text { IC a } 95 \%\end{array}$ & $\begin{array}{c}7,68 \pm 3.94 \\
5,8-9,5\end{array}$ & \\
\hline
\end{tabular}

d.p. desvio padrão; IC intervalo de confiança

Tabela 2 - Fiabilidade teste-reteste na amostra 1

\begin{tabular}{|c|c|c|c|}
\hline Item & Em geral, eu acredito que... & Pearson & ICC \\
\hline 1 & sei identificar os aspetos dos cuidados a ter com a minha diabetes com os quais estou insatisfeito & 0,330 & 0,990 \\
\hline 2 & consigo atingir as metas relativas à minha diabetes & 0,330 & 0,988 \\
\hline 3 & $\begin{array}{l}\text { posso encontrar diferentes formas de ultrapassar os problemas para atingir as metas relativas } \\
\text { à minha diabetes }\end{array}$ & 0,330 & 0,986 \\
\hline 4 & consigo arranjar forma de me sentir melhor mesmo tendo diabetes & 0,330 & 1,000 \\
\hline 5 & sei como lidar de forma positiva com o stress relacionado com a diabetes & 0,330 & 0,992 \\
\hline 6 & posso pedir ajuda por ter e para tratar a diabetes sempre que necessito & 0,330 & 0,931 \\
\hline 7 & sei o que me ajuda a estar motivado/a para cuidar da minha diabetes & 0,186 & 0,989 \\
\hline 8 & $\begin{array}{l}\text { me conheço suficientemente bem para fazer as melhores escolhas para cuidar da minha } \\
\text { diabetes }\end{array}$ & 0,330 & 1,000 \\
\hline
\end{tabular}

pontuação dada em cada item da escala.

A média do score final obtido foi de $4,1 \pm 0,8$ com um intervalo de confiança a $95 \%$ de 3,9 a 4,3. Em termos de consistência interna, obteve-se um valor a de Cronbach de 0,871 . Os valores obtidos pelo DES-SF pelos vários valores das variáveis género e idade são apresentados na Tabela 5 :

Como se pode observar nesta Tabela, a distribuição por sexo, por idade ou por HbA1c não apresentou qualquer diferença estatisticamente significativa. Analisámos também a relação entre os valores obtidos pela escala DES-SF e o tempo de diagnóstico. O valor de correlação encontrado ( $\rho=-0,032 ; p=0,779$ ) demonstra não haver correlação entre estas duas variáveis.

\section{DISCUSSÃO}

Após o trabalho de tradução e sua validação em 2013 pelo Centro de Estudos e Investigação em Saúde da Universidade de Coimbra (CEISUC) do original Diabetes
Empowerment Scale - Short Form (DES-SF), a primeira fase deste estudo teve como objetivo avaliar a fiabilidade desta escala de capacitação da diabetes. A amostra obtida para avaliar este parâmetro é relativamente envelhecida, com uma distribuição relativamente similar, no que diz respeito ao género e, com uma formação, na sua maioria, até à escolaridade básica.

Para conseguir aferir este parâmetro, foi usado o valor de alfa de Chronbach, coeficiente que mede a consistência interna de uma escala, tendo sido encontrado o valor 0,871 considerado muito bom. Procedeu-se também a um teste-reteste onde foram encontrados coeficientes de correlação de 0,330 , revelando a estabilidade temporal do DES-SF.

Procedeu-se depois a nova aplicação, sempre após a consulta de seguimento de enfermagem, em duas semanas, uma em janeiro e uma em fevereiro de 2014. Pela análise feita aos resultados obtidos, esta amostra parece-se com o observado em cuidados de saúde primários, ou seja uma população mais idosa, com um nível básico de 
Tabela 3 - Dados sociodemográficos e clínicos da amostra 2

\begin{tabular}{llcr}
\hline Variável & Valores & $\mathbf{n}$ & \% \\
\hline Amostra & & $\mathbf{8 1}$ & $\mathbf{1 0 0 , 0}$ \\
Género & Masculino & 45 & 55,6 \\
& Feminino & 36 & 44,4 \\
Idade (anos) & Média \pm d.p. & $68,5 \pm 10,3$ & $66,2-70,8$ \\
& IC a 95\% & 3 & 3,7 \\
Habilitações Literárias & Não sabe ler nem escrever & 17 & 66,7 \\
& Sabe ler e escrever & 54 & 6,2 \\
& Ensino básico $\left(6^{\circ}\right.$ ano) & 5 & 2,5 \\
Hba1c & Ensino Secundário $\left(12^{\circ}\right.$ ano) & 2 & $6,8 \pm 1,3$ \\
Tempo de diagnóstico & Ensino Superior & $6,5 \pm 7,1$ \\
& Média \pm d.p. & $9,2 \pm 8,0$ \\
\hline
\end{tabular}

d.p. desvio padrão; IC intervalo de confiança

Tabela 4 - Distribuição dos itens do DES-SF

\begin{tabular}{|c|c|c|c|c|c|c|c|c|c|c|}
\hline \multirow[t]{2}{*}{ Item } & \multicolumn{2}{|c|}{$\begin{array}{c}\text { Discordo } \\
\text { completamente } \\
\text { [1] }\end{array}$} & \multicolumn{2}{|c|}{$\begin{array}{l}\text { Discordo } \\
\text { um pouco } \\
\text { [2] }\end{array}$} & \multicolumn{2}{|c|}{$\begin{array}{l}\text { Nem discordo nem } \\
\text { concordo } \\
\text { [3] }\end{array}$} & \multicolumn{2}{|c|}{$\begin{array}{c}\text { Concordo } \\
\text { um pouco } \\
\text { [4] }\end{array}$} & \multicolumn{2}{|c|}{$\begin{array}{c}\text { Concordo } \\
\text { completamente } \\
\text { [5] }\end{array}$} \\
\hline & $\mathbf{n}$ & $\%$ & $n$ & $\%$ & $\mathbf{n}$ & $\%$ & $n$ & $\%$ & $\mathbf{n}$ & $\%$ \\
\hline 1 & 2 & 2,5 & 7 & 8,6 & 11 & 13,6 & 26 & 32,1 & 33 & 40,7 \\
\hline 2 & 1 & 1,2 & 7 & 8,6 & 12 & 14,8 & 26 & 32,1 & 35 & 43,2 \\
\hline 3 & 2 & 2,5 & 4 & 4,9 & 11 & 13,6 & 35 & 43,2 & 29 & 35,8 \\
\hline 4 & 4 & 4,9 & 3 & 3,7 & 3 & 3,7 & 22 & 27,2 & 40 & 49,4 \\
\hline 5 & 5 & 6,2 & 8 & 9,9 & 9 & 11,1 & 29 & 35,8 & 30 & 37,0 \\
\hline 6 & 1 & 1,2 & 7 & 8,6 & 2 & 2,5 & 19 & 23,5 & 52 & 64,2 \\
\hline 7 & 3 & 3,7 & 3 & 3,7 & 14 & 17,3 & 28 & 34,6 & 33 & 40,7 \\
\hline 8 & 2 & 2,5 & 3 & 3,7 & 10 & 12,3 & 24 & 29,6 & 42 & 51,9 \\
\hline
\end{tabular}

Tabela 5 - Distribuição do DES-SF

\begin{tabular}{|c|c|c|c|c|}
\hline Variável & Valores & Média & d.p. & Sig. \\
\hline Género & $\begin{array}{l}\text { Masculino } \\
\text { Feminino }\end{array}$ & $\begin{array}{l}4,12 \\
4,07\end{array}$ & $\begin{array}{l}0,84 \\
0,65\end{array}$ & 0,782 \\
\hline Idade (anos) & $\begin{array}{c}<50 \text { anos } \\
50-64 \text { anos } \\
65-74 \text { anos } \\
\geq 75 \text { anos }\end{array}$ & $\begin{array}{l}4,12 \\
4,01 \\
4,28 \\
3,91\end{array}$ & $\begin{array}{l}0,18 \\
0,86 \\
0,64 \\
0,81\end{array}$ & 0,331 \\
\hline $\mathrm{HbA1c}$ & $\begin{array}{l}<6,5 \% \\
\geq 6,5 \%\end{array}$ & $\begin{array}{l}4,08 \\
4,11\end{array}$ & $\begin{array}{l}0,69 \\
0,83\end{array}$ & 0,861 \\
\hline
\end{tabular}

instrução académica pela $4^{\mathrm{a}}$ classe antiga, hoje comparável ao $6^{\circ}$ ano. $\mathrm{O}$ nível de $\mathrm{HbA} 1 \mathrm{c}$, obtido pela média das duas últimas medições, foi de $6,8 \%$, valor quase idêntico $(6,9 \%)$ à média por utente com pedidos registados no SNS, em 2012. ${ }^{1}$ O tempo médio decorrido desde o diagnóstico (9,2 anos) significa que estamos perante uma doença de longa duração, verificando-se que, na maioria dos casos, os inquiridos atribuíram pontuações acima dos 4 pontos às afirmações que Ihes eram apresentadas, ou seja, concordando um pouco ou completamente com o que lhes era apresentado. A média do score final obtida, juntando as pontuações dos 8 itens da escala foi $4,1 \pm 0,8$, sendo um valor que é semelhante às médias obtidas na aplicação desta escala em vários estudos. ${ }^{6-9}$ Não houve diferença estatisticamente significativa entre a média do score final e o género $(p=0,780)$, a instrução $(p=0,119)$, a 
idade $(p=0,402)$ e o tempo de diagnóstico $(p=0,779)$. Entre o valor de HbA1c e a pontuação média do DES-SF verifica-se uma correlação positiva fraca $(\rho=-0,114 ; p=$ 0,312 ) o que sugere que estas duas variáveis fluem de forma quase harmoniosa, o que vai ao encontro do que é referido em outros artigos, assim se podendo perceber que, neste estudo, menor valor de médio de HbA1c está de acordo com respostas mais consistentes com melhor capacitação. $2,5-7,10$

Os resultados obtidos permitem-nos pensar que, pelo menos para esta amostra, o conhecimento relativo à boa compreensão e ao que mais corretamente fazer para manter a diabetes tipo 2 controlada está presente. ${ }^{2}$ De facto, é hoje ponto central que a capacitação é fonte da melhor adesão à terapêutica e à manutenção em bom estado de saúde. ${ }^{11,12}$

Utilizámos o valor de $\mathrm{HbA1c}$ médio mesmo sabendo que tal não serve hoje para aferição de bom controlo pois se para os jovens diabéticos que vão durar muito tempo em doença os níveis abaixo de 6,5\% são importantes, já para os idosos um valor mais elevado pode ser admitido até evitar complicações como a temida hipoglicémia. ${ }^{13}$ Acresce que será ainda mais importante o perfil glicémico ao longo do dia evitando excessivos picos glicémicos para se ajuizar de um bom controlo da pessoa com diabetes. ${ }^{13}$

Mesmo assim, a correlação obtida é importante pois permite-nos perceber que capacitação e o eventual melhor controlo da diabetes se verificam o que, para quem trabaIha com pessoas com diabetes como um todo é importante, podendo até pensar-se na criação de um indicador com-

\section{REFERÊNCIAS}

1. Gardete Correia L, Boavida JM, Fragoso de Almeida JP, Massano Cardoso S, Dores J, Sequeira Duarte J, et al. Diabetes: Factos e Números 2013 - Relatório Anual do Observatório Nacional da Diabetes. Lisboa: Sociedade Portuguesa de Diabetologia; 2013.

2. Leksell J, Funnell M, Sandberg G, Smide B, Wiklund G, Wikblad K. Psychometric properties of the Swedish Diabetes Empowerment Scale. Scand J Caring Sci. 2007;21:247-52.

3. Anderson RM, Funnell MM, Butler PM, Arnold MS, Fitzgerald JT, Feste CC. Patient empowerment. Results of a randomized controlled trial. Diabetes Care. 1995;18:943-9.

4. Anderson RM, Funnell MM, Fitzgerald JT, Marrero DG. The Diabetes Empowerment Scale: a measure of psychosocial self-efficacy. Diabetes Care. 2000;23:739-43.

5. Anderson RM, Fitzgerald JT, Gruppen LD, Funnell MM, Oh MS. The Diabetes Empowerment Scale-Short Form (DES-SF). Diabetes Care. 2003;26:1641-2.

6. Castillo A, Giachello A, Bates R, Concha J, Ramirez V, Sanchez C, et al. Community-based diabetes education for latinos: the Diabetes Empowerment Education Program. Diabetes Educator. 2010;36:58694.

7. Sperl-Hillen J, Beaton S, Fernandes O, Von Worley A, Vazquez-Benitez $\mathrm{G}$, Parker $\mathrm{E}$, et al. Comparative effectiveness of patient education posto para o seguimento de tal pessoa, que poderá incluir vertentes quanto a processo dos cuidados, a adequação técnico-científica, aos resultados, à capacitação e à eficiência.

Esta escala permite, assim, sabendo-se quais as piores respostas, transmitir conhecimento, mostrar onde ele está e aumentar a possibilidade de adequadas escolhas para o melhor controlo.

\section{CONCLUSÃO}

A Escala de Capacitação da Diabetes - Versão Breve (DES-SF) revelou ser uma escala fiável para medir a capacitação em doentes diabéticos em Portugal. Além disto, verificou-se uma correlação positiva entre o valor médio da DES-SF e os níveis de HbA1c.

\section{AGRADECIMENTOS}

Aos enfermeiros das USF Marquês de Marialva, Topázio e $\mathrm{S}$. Julião bem como aos respetivos coordenadores e conselhos técnicos, pela colaboração. Aos pacientes que, com consentimento, responderam ao questionário.

\section{CONFLITOS DE INTERESSE}

Os autores declaram não ter qualquer conflito de interesses para este trabalho.

\section{FONTES DE FINANCIAMENTO}

Este estudo foi realizado apenas a expensas dos autores.

methods for type 2 diabetes: a randomized controlled trial. Arch Intern Med. 2011;171:2001-10.

8. Tang TS, Funnell MM, Noorulla S, Oh M, Brown MB. Sustaining shortterm improvements over the long-term: results from a 2-year diabetes self-management support (DSMS) intervention. Diabetes Res Clin Pract. 2012;95:85-92.

9. Tang TS, Funnell MM, Brown MB, Kurlander JE. Self-management support in "real-world" settings: an empowerment-based intervention. Patient Educ Couns. 2010;79:178-84.

10. Shiu AT, Wong RY, Thompson DR. Development of a reliable and valid Chinese version of the diabetes empowerment scale. Diabetes Care. 2003;26:2817-21.

11. Little P, Everitt H, Williamson I, Warner G, Moore M, Gould C, et al Observational study of effect of patient centredness and positive approach on outcomes of general practice consultations. BMJ. 2001;323:908-11.

12. Howie JG, Heaney D, Maxwell M. Quality, core values and the general practice consultation: issues of definition, measurement and delivery. Fam Pract. 2004;21:458-68.

13. Duarte R, Silva Nunes J, Dores J, Medina JL, Ruas A, Coelho A, et al. Recomendações Nacionais da SPD para o Tratamento da Hiperglicemia na Diabetes Tipo 2. Rev Port Diabetes. 2013:8:4-29. 
Marcelo AVEIRO, Luiz Miguel SANTIAGO, Pedro LOPES FERREIRA, José Augusto SIMÕES

\section{Estudo de Fiabilidade da Escala de Capacidade de Controlo da Diabetes: Versão Breve}

Acta Med Port 2015:28:177-181

Publicado pela Acta Médica Portuguesa, a Revista Científica da Ordem dos Médicos

Av. Almirante Gago Coutinho, 151

1749-084 Lisboa, Portugal.

Tel: +351218428 215

E-mail: submissao@actamedicaportuguesa.com

www.actamedicaportuguesa.com

ISSN:0870-399X | e-ISSN: 1646-0758

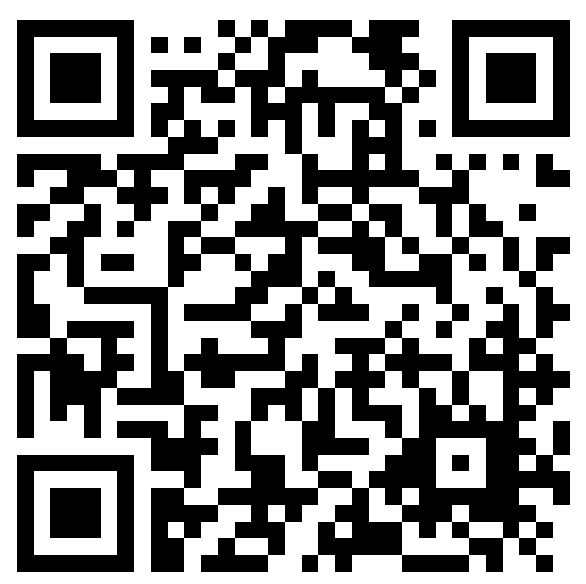

